\title{
Late Diagnosis of COVID-19 in Patients Admitted to the Hospital
}

J Gen Intern Med 35(9):2829-31

DOI: $10.1007 / \mathrm{s} 11606-020-05949-1$

(C) Society of General Internal Medicine 2020

\section{BACKGROUND}

Testing for severe acute respiratory syndrome coronavirus 2 (SARS-CoV-2) is vital for identification of cases. Currently, the Centers for Disease Control and Prevention recommends that hospitalized patients with symptoms receive priority for testing. ${ }^{1}$

Delayed testing could occur because patients have atypical presentations or the initial test is a false negative. Since test sensitivity depends on the site sampled ${ }^{2}$ and hospital protocols usually rely on nasal samples, patients with lower respiratory tract infections can have a false-negative result. ${ }^{3}$

Understanding how often the diagnosis of SARS-CoV-2 is missed is essential for infection control. We describe the frequency and characteristics of hospitalized patients with COVID-19 diagnosed after admission.

\section{METHODS}

This retrospective cohort study included adults hospitalized before April 20, 2020, who tested positive for SARS-CoV-2. Testing became available for Cleveland Clinic Health System (CCHS) patients with suspected COVID-19 starting March 6 and was restricted to symptomatic, high-risk patients (e.g., hospitalized patients) on March 10. We identified patients with a positive SARS-CoV-2 test through CCHS's COVID-19 registry. We compared patients whose first positive SARS-CoV-2 test was on the day of admission versus later ("late diagnosis").

We collected demographic and health data from our electronic health record and the registry. We collected common symptoms of COVID-19. We also created a binary indicator of having none of the cardinal symptoms of COVID-19: cough, shortness of breath, or fever.

We report descriptive statistics. To identify factors associated with late diagnosis, we used a multivariable logistic regression model that included presenting symptoms,

Elizabeth R. Pfoh and Essa H. Hariri contributed equally to this work.

Received May 20, 2020

Accepted May 28, 2020

Published online June 15, 2020 comorbidities, age, and sex. We used model building techniques to identify variables that were associated with late diagnosis and picked the model with the lowest AIC and BIC. To assess the association between late diagnosis and length of stay, intensive care unit (ICU) admission, discharge disposition, and mortality, we conducted multivariable regressions that included age, sex, and the variables associated with late diagnosis. CCHS's IRB deemed this study exempt.

\section{RESULTS}

Our study included 356 patients; $86 \%$ with COVID-19 diagnosed on admission and $14 \%$ had a late diagnosis $(n=49)$. Patients diagnosed later presented without fever, shortness of breath, or cough $29 \%$ of the time (Table 1); $86 \%$ of the time they had $\geq 1$ common symptom. They were similar in age, sex, and pre-existing comorbidities to patients diagnosed on admission. In the adjusted model, patients with late diagnosis presented less often with sputum (AOR 0.26 , 95\% CI 0.08 0.88 ) and more often with heart failure (AOR 2.36, 95\% CI 1.15-4.84) and with none of the cardinal symptoms (AOR $2.89,95 \%$ CI 1.30-6.43). Late diagnosis was not associated with length of stay, ICU admission, death, or discharge home (Table 2).

Eleven patients with late diagnosis had an initial negative result and 4 had two prior negative results. Four of 11 had their positive result collected from a non-nasal site. False negatives averaged 3.7 days to a positive result versus 2.2 days for others with late diagnosis $(P>0.05)$. False negatives were retested due to worsening symptoms (7/11), imaging suggestive of COVID-19 (2/11), and discharge protocol for skilled nursing facility $(2 / 11)$.

\section{DISCUSSION}

Undiagnosed patients with COVID-19 can increase caregivers' risk of infection, as protective equipment may not be used. We found that $14 \%$ of COVID-19 patients were diagnosed after admission and most presented with $\geq 1$ common symptom. Few late diagnoses were due to a false-negative test. Retesting of false negatives often occurred after clinical deterioration, but 2 patients were not diagnosed until discharge. This study is limited because we could only identify false negatives if a patient was retested. Further, we could only include routinely collected clinical data. In conclusion, routine 
Table 1 Demographic and Presenting Characteristics of Hospitalized Patients with COVID-19

\begin{tabular}{|c|c|c|c|}
\hline & At hospitalization & After hospitalization & $P$ value $^{\mathbf{a}}$ \\
\hline$N$ & 307 & 49 & \\
\hline \multicolumn{4}{|l|}{ Demographics } \\
\hline \multicolumn{3}{|l|}{ Race $(\%)$} & \multirow[t]{5}{*}{0.36} \\
\hline Asian & $5(2 \%)$ & $0(0 \%)$ & \\
\hline Black & $112(36 \%)$ & $22(45 \%)$ & \\
\hline Other & $15(5 \%)$ & $4(8 \%)$ & \\
\hline White & $175(57 \%)$ & $23(47 \%)$ & \\
\hline Male $(\%)$ & $176(57 \%)$ & $27(55 \%)$ & 0.77 \\
\hline Hispanic $(\%)$ & $11(4 \%)$ & $5(11 \%)$ & 0.03 \\
\hline \multicolumn{3}{|l|}{ Smoking (\%) } & \multirow[t]{4}{*}{0.05} \\
\hline Current smoker & $25(8 \%)$ & $2(4 \%)$ & \\
\hline Former smoker & $115(38 \%)$ & $20(43 \%)$ & \\
\hline Non-smoker & $146(49 \%)$ & $25(53 \%)$ & \\
\hline Age (mean [SE]) & $66.0(0.9)$ & $67.9(2.2)$ & 0.47 \\
\hline \multicolumn{4}{|l|}{ Exposure history } \\
\hline Exposed to COVID-19 & $82(28 \%)$ & $7(16 \%)$ & 0.35 \\
\hline Family member w/ COVID-19 & $38(13 \%)$ & $3(7 \%)$ & 0.23 \\
\hline \multicolumn{4}{|l|}{ Presenting symptoms } \\
\hline Cough $(\%)$ & $226(74 \%)$ & $27(55 \%)$ & 0.01 \\
\hline Fever $(\%)$ & $158(51 \%)$ & $22(45 \%)$ & 0.39 \\
\hline Shortness of breath (\%) & $195(64 \%)$ & $23(47 \%)$ & 0.03 \\
\hline Two or more of cardinal symptoms of COVID $-19^{b}$ & $209(68 \%)$ & $29(59 \%)$ & 0.22 \\
\hline None of the cardinal symptoms of COVID- $19^{\mathrm{b}}$ & $36(12 \%)$ & $14(29 \%)$ & $<0.01$ \\
\hline Fatigue $(\%)$ & $167(54 \%)$ & $19(39 \%)$ & 0.04 \\
\hline Sputum production $(\%)$ & $74(23 \%)$ & $4(8 \%)$ & 0.02 \\
\hline Flu-like symptoms (\%) & $164(53 \%)$ & $21(43 \%)$ & 0.17 \\
\hline Diarrhea $(\%)$ & $92(30 \%)$ & $11(22 \%)$ & 0.28 \\
\hline Loss of appetite $(\%)$ & $95(31 \%)$ & $12(24 \%)$ & 0.36 \\
\hline Vomiting $(\%)$ & $37(12 \%)$ & $1(2 \%)$ & 0.04 \\
\hline At least one presenting symptom & $287(93 \%)$ & $42(86 \%)$ & 0.06 \\
\hline \multicolumn{4}{|l|}{ Comorbidities } \\
\hline BMI (mean [SE])* & $30.4(0.42)$ & $30.2(1.1)$ & 0.85 \\
\hline COPD/emphysema & $47(17 \%)$ & $6(14 \%)$ & 0.34 \\
\hline Asthma & $63(23 \%)$ & $12(30 \%)$ & 0.35 \\
\hline Diabetes & $125(45 \%)$ & $16(36 \%)$ & 0.26 \\
\hline Hypertension & $227(78 \%)$ & $35(78 \%)$ & 0.94 \\
\hline Coronary artery disease & $62(23 \%)$ & $11(27 \%)$ & 0.58 \\
\hline Heart failure & $68(25 \%)$ & $16(39 \%)$ & 0.06 \\
\hline Cancer & $53(18 \%)$ & $12(27 \%)$ & 0.19 \\
\hline Transplant history & $13(5 \%)$ & $0(0 \%)$ & 0.15 \\
\hline Connective tissue disease & $49(18 \%)$ & $8(20 \%)$ & 0.79 \\
\hline Inflammatory bowel disease & $16(6 \%)$ & $3(7 \%)$ & 0.76 \\
\hline Immunosuppressive disease & $69(24 \%)$ & $13(30 \%)$ & 0.29 \\
\hline \multicolumn{4}{|l|}{ Vaccination history } \\
\hline Flu shot & $158(51 \%)$ & $28(57 \%)$ & 0.46 \\
\hline Pneumovax shot & $103(34 \%)$ & $20(41 \%)$ & 0.32 \\
\hline \multicolumn{4}{|l|}{ Home medications } \\
\hline NSAIDS & $105(34 \%)$ & $20(41 \%)$ & 0.37 \\
\hline Steroids & $46(15 \%)$ & $10(20 \%)$ & 0.33 \\
\hline Carvedilol & $17(6 \%)$ & $3(6 \%)$ & 0.87 \\
\hline ACE inhibitor & $39(13 \%)$ & $11(22 \%)$ & 0.07 \\
\hline ARB & $35(11 \%)$ & $5(10 \%)$ & 0.81 \\
\hline Melatonin & $19(6 \%)$ & $13(27 \%)$ & $<0.01$ \\
\hline \multicolumn{4}{|l|}{ Hospital transfer } \\
\hline Hospital transfer & $3(1 \%)$ & $5(10 \%)$ & $<0.01$ \\
\hline
\end{tabular}

*Body mass index (BMI) missing in 11 (3\%) of respondents; presenting symptoms, comorbidities, vaccination history, and home medications are shown if they were positively identified

Presenting symptoms, comorbidities, vaccination history, and home medic ations are shown if they were positively identified

${ }^{a} P$ values were obtained using chi-square and Student's T tests

${ }^{b}$ Cardinal symptoms of COVID-19 include cough, shortness of breath, and fever 
Table 2 Outcomes for Patients Hospitalized with COVID-19

\begin{tabular}{|c|c|c|c|c|c|}
\hline & \multicolumn{2}{|c|}{ At hospitalization } & \multicolumn{2}{|c|}{ After hospitalization } & \multirow[t]{2}{*}{ Adjusted $P$ value $^{a}$} \\
\hline & $N$ & \%/median (IQR) & $N$ & \%/median (IQR) & \\
\hline \multicolumn{6}{|l|}{ Patient outcomes } \\
\hline ICU admission & 111 & $36 \%$ & 23 & $47 \%$ & 0.15 \\
\hline Died & 46 & $16 \%$ & 10 & $23 \%$ & 0.51 \\
\hline Length of stay (median [IQR]) & 239 & $4[2-8]$ & 38 & $6[3-12]$ & 0.16 \\
\hline Discharge home (versus other) & 153 & $63 \%$ & 27 & $71 \%$ & 0.09 \\
\hline
\end{tabular}

Missing death information on 24 people (7\%); missing length of stay and discharge destination on 79 people (22\%)

$I Q R$ interquartile range

${ }^{a} P$ values were obtained using an adjusted regression model including the following confounders: age, sex, heart failure, sputum on admission and binary indicator of no cough, shortness of breath, or fever on admission

testing on admission may reduce delayed identification of COVID-19. Retesting is also warranted.

Elizabeth R. Pfoh, $P h D, M P H^{1}$

Essa H. Hariri, $M D^{2}$

Anita D. Misra-Hebert, $M D, M P H^{3}$

Abhishek Deshpande, $\mathrm{PhD}, \mathrm{MD}^{1}$

Lara Jehi, $M D^{4}$

Michael B. Rothberg, MD, MPH ${ }^{1}$

${ }^{1}$ Center for Value-Based Care Research, Cleveland Clinic Community Care,

9500 Euclid Avenue, Cleveland, OH 44195, USA

${ }^{2}$ Department of Internal Medicine, Cleveland Clinic Community Care,

Cleveland, OH, USA

${ }^{3}$ Healthcare Delivery and Implementation Science Center, Cleveland Clinic,

Cleveland, OH, USA

${ }^{4}$ Chief Research Information Officer, Cleveland Clinic, Cleveland, OH, USA

Corresponding Author: Elizabeth R. Pfoh, PhD, MPH; Center for Value-Based Care Research, Cleveland Clinic Community Care, 9500 Euclid Avenue, Cleveland, OH 44195, USA (e-mail: pfohe@ccf.org).
Author Contributions All authors contributed to the study design. $L J$ and EH collected the data. EP conducted the data analysis and drafted the manuscript. All authors revised the manuscript for important intellectual content.

\section{Compliance with Ethical Standards:}

Conflict of Interest: The authors declare that they do not have a conflict of interest. Dr. Misra-Hebert reports funding from the Agency for Healthcare Research and Quality KO8 HSO24128 and reports grants from NHLBI, grants from Novo Nordisk, Inc, grants from Merck Inc., grants from Boehringer Ingelheim Pharmaceuticals, Inc, outside the submitted work. Dr. Abhishek Deshpande has received research support from Clorox Healthcare not related to this study and is on the advisory board for Ferring Pharmaceuticals.

\section{REFERENCES}

1. Centers for Disease Control and Prevention. Evaluating and Testing Persons for Coronavirus Disease 2019 (COVID-19). Available at: https:// www.cdc.gov/coronavirus/2019-nCoV/hcp/clinical-criteria.html. Accessed May 8, 2020

2. Wang W, Xu Y, Lu R, et al. Detection of SARS-CoV-2 in Different Types of Clinical Specimens. JAMA. 2020 Mar 11. doi: https://doi.org/10.1001/ jama.2020.3786. [Epub ahead of print]

3. Xie, Xingzhi, Zhong $\mathbf{Z}$, Zhao w, et al. Chest CT for typical 2019-nCoV pneumonia: relationship to negative RT-PCR testing. Radiology (2020): 200343.

Publisher's Note: Springer Nature remains neutral with regard to jurisdictional claims in published maps and institutional affiliations. 